\title{
A WIRELESS MIMO CHANNEL PROBING APPROACH FOR ARBITRARY ANTENNA ARRAYS
}

\author{
Brian D. Jeffs, Edward Pyper,and Brandon Hunter \\ Brigham Young University, Department of Electrical and Computer Engineering \\ 459 CB, Provo, UT 84602, email: bjeffs@ee.byu.edu
}

\begin{abstract}
This paper introduces a method for experimentally probing indoor wireless channels to compute MIMO transfer function matrices, $\mathbf{H}$. The data is gathered using rotating transmit and receive antennas to observe the multipath ray structure of the channel. $\mathbf{H}$ can be computed for any arbitrary transmit and receive array sizes and geometries using the single probe data set. Estimating $\mathbf{H}$ is crucial for spacetime coding communications algorithm performance analysis. Example results are presented.
\end{abstract}

\section{INTRODUCTION}

With the promise of dramatic capacity improvements, space time coding and related MIMO wireless communications systems have become important areas of research and development activity [1] [2]. Such approaches depend on rich scattering environments and knowledge of the channel transfer matrix at the receiver, transmitter, or both. A crucial step in developing such systems is forming realistic channel models for use in statistical algorithm performance analysis. At the present time, very few experiments have been reported where MIMO channel characteristics have been measured in practical environments. The issues of determining where space time coding will work, and finding optimal array geometries for highest capacity gains have not been fully resolved. Answering these questions will require a wide range of MIMO channel probing measurements with a variety of array geometries and locations, including indoor, outdoor, fixed and mobile environments.

Channel probing is expensive, time consuming, and impractical for the large variety of array geometries that need to be evaluated for space-time coding applications. An approach is needed which uses a common set of probing data from a single experiment to compute the MIMO channel matrix, $\mathbf{H}$,for any arbitrary proposed array geometry without needing to take new field measurements. Current experiments by our own group and others ( [3], Lucent, AT\&T

This project was supported by National Science Foundation grant number CCR-9979452.
Laboratories, etc.) are being undertaken to physically measure $\mathbf{H}$ for a specific transmitter - receiver array pair. However, the proposed ray based approach described below is the only one we are aware of which can generate a measured $\mathbf{H}$ for an array whose geometry is specified after probing experiment data collection.

\subsection{Channel Ray Model}

We will assume propagation in the horizontal plane and flat fading. The channel model is based on Spencer's [4], which is an extension of [5]. To the Spencer statistical model we add a ray departure angle, $\theta$, and drop the time of arrival terms since we are dealing with narrowband flat fading communications. The channel is represented as a sum of discrete ray paths:

$$
C(\phi, \theta) .=\sum_{k=1}^{K} \beta_{k} e^{j \psi_{k}} \delta\left(\phi-\Phi_{k}, \theta-\Theta_{k}\right)
$$

where $\Phi_{k}$ and $\Theta_{k}$ are the departure and arrival angles respectively for the $k^{\text {th }}$ ray in the channel from transmit to receive array. As in [4], these are random variables with a mixture of uniform and Laplacian distributions based on a cluster model. $\beta_{k}$ is Rayleigh distributed with a random mean drawn from a double exponential distribution, and $\psi_{k}$ is uniform $(0,2 \pi)$.

The MIMO narrowband transfer matrix, $\mathbf{H}$, relates the complex transmit array excitation vector, a, to the observed receiver array element response vector, $\mathbf{y}$, as $\mathbf{y}=\mathbf{H a}$. Using the individual ray parameters from the $C(\phi, \theta)$ model, one can compute $\mathbf{H}$ for any given transmit and receive array configuration.

$$
\begin{aligned}
\mathbf{H}= & \sum_{k=1}^{K} \beta_{k} e^{j \psi_{k}}\left[\mathbf{d}_{r}\left(\Theta_{k}\right) \odot \mathbf{v}_{r}\left(\Theta_{k}\right)\right] \\
& \times\left[\mathbf{d}_{t}\left(\Phi_{k}\right) \odot \mathbf{v}_{t}\left(\Phi_{k}\right)\right]^{T} \\
\mathbf{d}_{r}(\theta)= & {\left[d_{r_{1}}(\theta), \cdots d_{r_{L}}(\theta)\right]^{T} } \\
\mathbf{v}_{r}(\theta)= & {\left[e^{j \frac{\omega}{c} \vec{r}_{1} \cdot \vec{s}_{\theta}}, \cdots e^{j \frac{\omega}{c} \vec{r}_{L} \cdot \vec{s}_{\theta}}\right]^{T} } \\
\vec{s}_{\theta}= & \hat{i} \cos \theta+\hat{j} \sin \theta
\end{aligned}
$$


where ' $\odot$ ' indicates the element-wise Schur matrix product, ' $X$ ' is the conventional matrix product, and '. ' is the inner product of 3-D vectors. $\vec{t}_{l}$ and $\vec{r}_{l}$ are the 3 -D position vectors for the $l^{\text {th }}$ elements of the transmit and receive arrays respectively. $d_{r_{l}}(\theta)$ is the directional response of the $l^{\text {th }}$ element, $\mathbf{v}_{r}(\phi)$ is the receive array steering vector for direction $\theta$ (i.e array response to a unit plane wave from direction $\theta$ ), and $\hat{i}$ and $\hat{j}$ are $x$ and $y$ unit vectors respectively. $\mathbf{v}_{t}(\phi), \vec{s}_{\phi}$, and $\mathbf{d}_{t}(\phi)$ are defined similarly.

\section{A BI-DIRECTIONAL CHANNEL PROBER}

\subsection{Experiment Description}

Figure 1 is a photograph of the probing test bed used for indoor $\mathbf{H}$ estimation with arbitrary arrays. We refer to this system as the "bi-directional channel probe" because measurements are taken while rotating directional antennas at both the transmit and receive ends. The $22 \mathrm{~cm}$ square horn antenna on the right is used to transmit a $6.0 \mathrm{GHz} \mathrm{CW}$ tone. The $61 \mathrm{~cm}$ parabolic dish antenna on the left is used to receive signals. Antenna $3 \mathrm{~dB}$ beamwidths are approximately 10 and 3 degrees respectively. A computer on the receiver cart controls a Hewlett Packard microwave network analyzer which generates the probe r.f. signal. This is then amplified and sent by a 200 foot coax cable to the remote transmit horn. Under computer control, the transmit horn is stepped through 36 discrete angles, $\phi_{m}$, at $10^{\circ}$ increments. For each $\phi_{m}$ setting, the receiver dish is stepped through 540 discrete angles, $\theta_{n}$, covering $360^{\circ}$. The dish signal is amplified and fed back to the network analyzer input. The analyzer calculates a magnitude and phase response for the entire transmit - receive loop. Calibration responses for the amplifiers, cables, and feeds are removed from each measurement. By recording received amplitudes and phases for all combinations of transmit departure and receive arrival angles, individual rays can be identified as corresponding to amplitude peaks, albeit blurred by the antenna beam response patterns.

Probing measurements may be modeled as

$$
\begin{aligned}
& P\left(\phi_{m}, \theta_{n}\right) \\
& =\iint_{-\pi}^{\pi} b_{t}\left(\phi-\phi_{m}\right) b_{r}\left(\theta-\theta_{n}\right) C(\phi, \theta) d \phi d \theta \\
& \quad+\eta\left(\phi_{m}, \theta_{n}\right) \\
& =\sum_{k=1}^{K} \beta_{k} e^{j \psi_{k}} b_{t}\left(\Phi_{k}-\phi_{m}\right) b_{r}\left(\Theta_{k}-\theta_{n}\right) \\
& \quad+\eta\left(\phi_{m}, \theta_{n}\right)
\end{aligned}
$$

where $b_{t}(\phi)$ and $b_{r}(\theta)$ are the transmit and receive probing beam responses respectively and $\eta$ is system noise. $\phi=0$, $\theta=0$ correspond to the mainlobe peaks. The goal of this work is to use probing measurements,

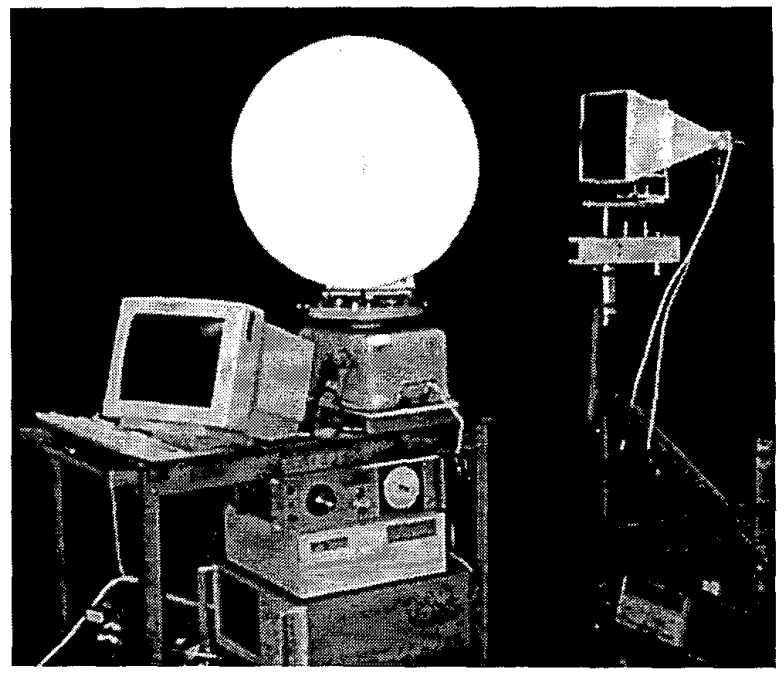

Fig. 1. Experimental channel probing apparatus.

$P\left(\phi_{m}, \theta_{n}\right)$, to form $\mathbf{H}$ estimates for any desired arbitrary set of transmit - receive antenna arrays located in the same general positions as the probing antennas. Given array element locations $\left(\vec{t}_{l}\right.$ and $\left.\vec{r}_{l}\right)$ and directional responses $\left(d_{t_{l}}(\phi)\right.$ and $d_{r_{l}}(\theta)$ ), we wish to compute $\mathbf{H}$ for a variety of arrays given a single data set $P\left(\phi_{m}, \theta_{n}\right)$.

We propose the following error metric to be used in evaluating algorithms for accuracy in estimating $\mathbf{H}$.

$$
\begin{aligned}
\epsilon & =\min _{\alpha} \frac{\|\mathbf{H}-\alpha \hat{\mathbf{H}}\|_{F}^{2}}{\|\mathbf{H}\|_{\boldsymbol{F}}^{2}} \\
\alpha_{\min } & =\frac{\hat{\mathbf{h}}^{H} \mathbf{h}}{\hat{\mathbf{h}}^{H} \hat{\mathbf{h}}}, \mathbf{h}=\operatorname{vec}\{\mathbf{H}\}
\end{aligned}
$$

This metric is insensitive to bulk scale and phase so that it is invariant to probing instrument calibration errors which do not affect channel capacity calculations. It is however sensitive to element wise deviations, which represent single transmit - receive antenna pairs. The normalized error metric ranges over $0 \leq \epsilon \leq 1$.

\subsection{A Deconvolution Approach to Channel Estimation}

The apparent difficulty with direct use of $P$ in $\mathbf{H}$ estimation is that the individual ray paths are not isolated for use in equation (2). As can be seen in equation (4), $P$ has the form of a 2-D convolution of scaled delta functions (corresponding to ray departure/arrival angle pairs) with a a blurring beam response function, $h[m, n]=b_{t}\left(\phi_{m}\right) b_{r}\left(\theta_{n}\right)$. What is required is a deconvolution method to recover an estimate of $C(\phi, \theta)$ through its parameters, $\beta_{k}, \psi_{k}, \Phi_{k}$, and $\Theta_{k}$. With these parameters, equation (2) can be directly evaluated to yield $\hat{\mathbf{H}}$. 


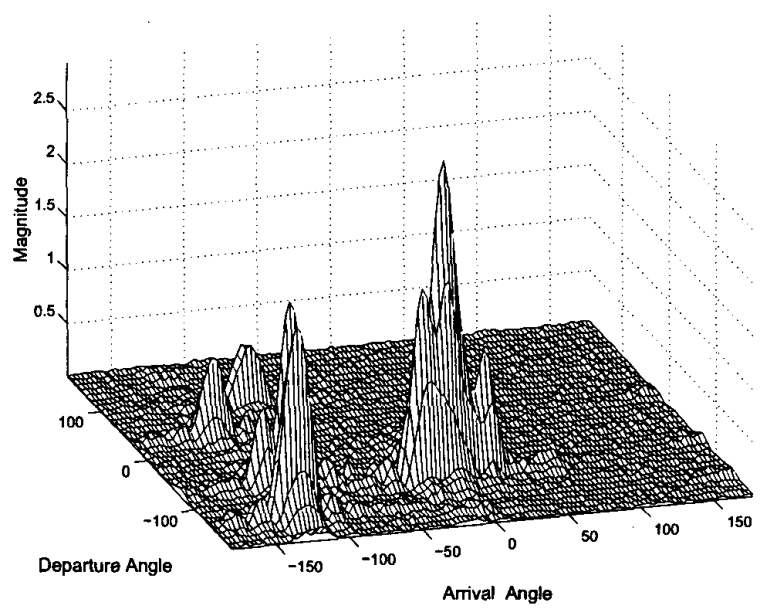

Fig. 2. Simulated channel probing data. Peak SNR is 40 $\mathrm{dB}$, the channel had 12 rays consisting of 4 clusters of 3 elements each.

We propose using a modification of the CLEAN algorithm [6], which is particularly well suited for deconvolving blurred point source images like this discrete ray angle map. This method, also referred to as "iterative beam subtraction," locates rays by finding the peak magnitude in $P\left(\phi_{m}, \theta_{n}\right)$, fitting a scaled, shifted copy of $h[m, n]$ to this peak, and subtracting it from $P$. This removes (approximately) the effect of one ray from $P$. The process is repeated to locate each successive ray until the residual magnitude drops below the noise or modeling error threshold. The specific algorithm used is described below.

\section{Ray Deconvolution Algorithm:}

1. Initialize the residual matrix: $k=1, R^{1}\left(\phi_{m}, \theta_{n}\right)=$ $P\left(\phi_{m}, \theta_{n}\right)$.

2. Find the peak: $M, N=\arg \max _{m, n}\left|R^{k}\left(\phi_{m}, \theta_{n}\right)\right|$.

3. Form residual vector around neighborhood of peak: $\mathbf{p}=\operatorname{vec}\left\{R^{k}\left(\phi_{m}, \theta_{n}\right)\right\}, \forall(m, n) \in \mathcal{N}\{(M, N)\}$.

4. Find the least squares fit to the residual peak for a shifted, complex scaled, 2-D probing beam response:

$$
\begin{aligned}
\alpha_{\mathrm{opt}}, \Delta_{\phi}, \Delta_{\theta}= & \arg \min _{\alpha, \delta_{\phi}, \delta_{\theta}}\left\|\mathbf{p}-\alpha \hat{\mathbf{p}}\left(\delta_{\phi}, \delta_{\theta}\right)\right\|^{2} \\
\hat{\mathbf{p}}\left(\delta_{\phi}, \delta_{\theta}\right)= & \operatorname{vec}\left\{b_{t}\left(\phi_{m}-\phi_{M}-\delta_{\phi}\right)_{(360)}\right. \\
& \times b_{r}\left(\theta_{n}-\theta_{N}-\delta_{\theta}\right)_{(360)} \\
& \forall(m, n) \in \mathcal{N}\{(M, N)\} \\
\alpha_{\text {opt }}= & \frac{\hat{\mathbf{p}}^{H} \mathbf{p}}{\hat{\mathbf{p}}^{H} \hat{\mathbf{p}}}
\end{aligned}
$$

5. Add ray parameters to the model $\hat{C}(\phi, \theta): \hat{\beta}_{k}=|\alpha|$, $\hat{\psi}_{k}=L \alpha, \hat{\Phi}_{k}=\phi_{M}+\Delta_{\phi}, \hat{\Theta}_{k}=\theta_{N}+\delta_{\theta}$.
6. Remove the ray peak from residual:

$$
\begin{aligned}
& R^{k+1}\left(\phi_{m}, \theta_{n}\right)=R^{k}\left(\phi_{m}, \theta_{n}\right) \\
& \quad-\alpha_{\text {opt }} b_{t}\left(\phi_{m}-\hat{\Phi}_{k}\right)_{(360)} b_{r}\left(\theta_{n}-\hat{\Theta}_{k}\right)_{(360)} .
\end{aligned}
$$

7. If $\max _{m, n}\left|R^{k}\left(\phi_{m}, \theta_{n}\right)\right|>T, k=k+1$, go to 2 .

8. Calculate $\hat{\mathbf{H}}$ using equation (2). Done.

Subscript (360) indicates modulo 360 degree periodic angle addition, and $\mathcal{N}\{(M, N)\}$ is the set of 2-D samples, $(m, n)$, in the neighborhood of $(M, N)$. We typically use a neighborhood width sufficient to contain the main lobes of $b_{t}$ and $b_{r}$.
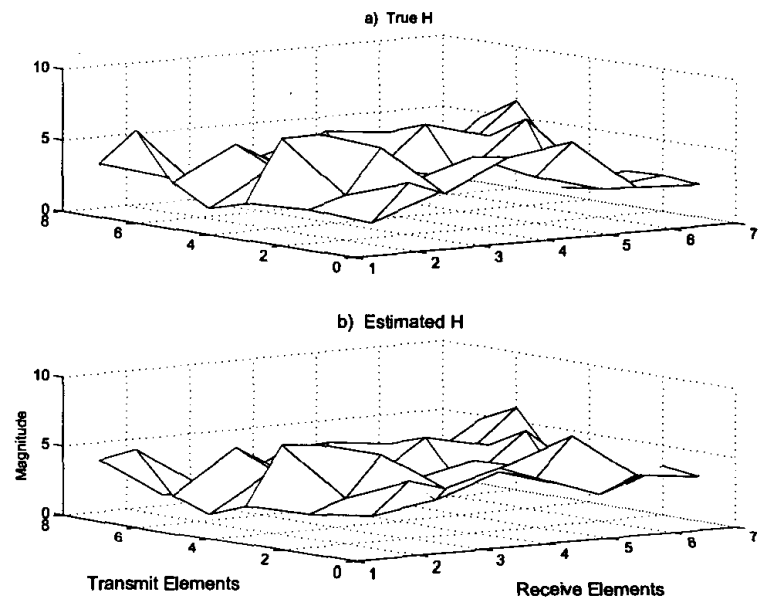

Fig. 3. a) True $\mathbf{H}$ matrix for simulated probing experiment. b) Estimated $\hat{\mathbf{H}}$. Transmit and receive arrays were 7 element ULAs with $5 \mathrm{~cm}$ element spacing.

\section{CHANNEL PROBING RESULTS}

\subsection{Synthetic Data Experiments for Validation}

In order to evaluate the effectiveness of the proposed algorithm, synthetic realizations of channel model(1) were generated. Channel parameters were drawn from random distributions consistent with indoor propagation in a reinforced concrete multistory building [4]. Synthetic probing measurements, $P\left(\phi_{m}, \theta_{n}\right)$ were then generated. $\hat{\mathbf{H}}$ was computed from $P$, and compared with the true $\mathbf{H}$ which was computed directly from $C(\phi, \theta)$. Figure 2 shows one of the probe realizations. Note that some ray peaks are blurred together and cover a range of departure and arrival angles. Probing beam responses were for $20 \mathrm{~cm}$ dish antennas at each end, with approximately 10 degree beamwidths operating at $6 \mathrm{GHz}$. 
Figure 3 shows the true and estimated $\mathbf{H}$ magnitudes corresponding to $P$ in Figure 2 for a 7 element ULAs with $5 \mathrm{~cm}$ element spacing: Note the striking similarity. The computed estimation error was $\epsilon=0.048$, which compares favorably with the error range of $0.7<\epsilon<.95$ found with other algorithms we evaluated.

\subsection{A Real-World Channel Probing Experiment}

Figure 4 shows probe data, $P\left(\phi_{m}, \theta_{n}\right)$,co llected by the experimental platform of Figure 1.

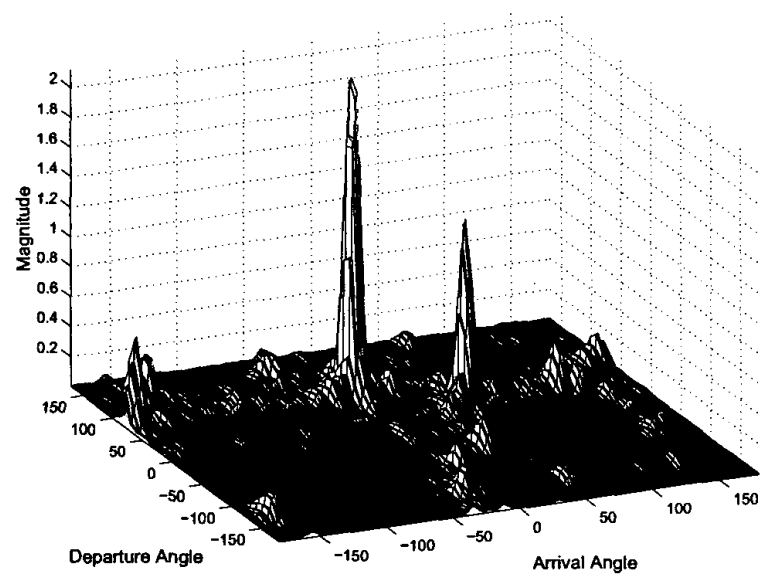

Fig. 4. Actual channel probing data. Both transmitter and receiver were located in the same 20 by $40 \mathrm{ft}$. room. Note the line of sight path is the highest peak.

Figure 5 shows $\hat{\mathbf{H}}$ computed for two different array geometries. Of note is the fact that the circular array matrix has 7 significant singular values, as compared with only 5 for the line array. The circular array would therefore support higher capacity space time processing. This illustrates the importance of evaluating a variety of array configurations at each probing site.

\section{CONCLUSIONS AND FUTURE WORK}

The validation experiments in Section 3.1 suggest the proposed algorithm produces reliable channel transfer matrix estimates. We are currently building a new test platform for a further verification experiment to directly measure $\mathbf{H}$ for specific arrays with up to 16 by 16 elements. These results will be compared with the $\hat{\mathbf{H}}$ estimated from probing platform described above. We may then draw more precise conclusions regarding accuracy of the present system for arbitrary arrays.
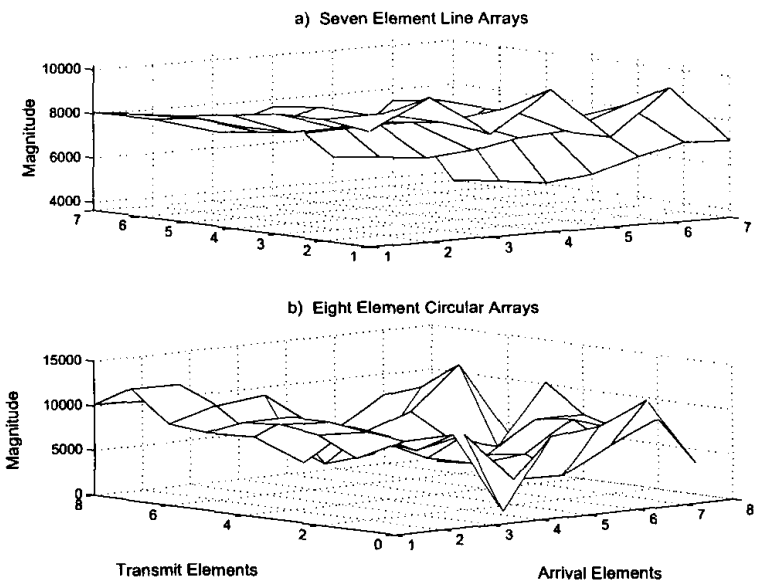

Fig. 5. Channel matrix estimates from real data. a) 7 element line arrays, $5 \mathrm{~cm}$ spacing. b) 8 element circular array, $20 \mathrm{~cm}$ diameter.

\section{REFERENCES}

[1] G.G. Raleigh and J.M. Cioffi, "Spatio-temporal coding for wireless communication," IEEE Transactions on Communications, vol. 46, no. 3, pp. 357-366, Mar. 1998 .

[2] G.D. Golden, C.J. Foschini, R.A. Valenzuela, and P.W. Wolniansky, "Detection algorithm and initial laboratory results using v-blast space-time communication architecture," Electronics Letters,v ol. 35, no. 1, Jan. 1999.

[3] M. Nilsson, B. Völcker, and B. Ottersten, "A cluster approach to spatio-temporal channel estimation," in IEEE International Conference on Acoustics, Speech, and Signal Processing, Istanbul, Turkey, Apr. 2000, vol. V, pp. 2757-2760.

[4] Q.H. Spencer, B.D. Jeffs, M.A. Jensen, and A.L. Swindlehurst, "Modeling the statistical time and angle of arrival characteristics of an indoor multipath channel," IEEE Journal on Selected Areas in Communications, vol. 18, no. 3, pp. 347-360, Mar.2 000.

[5] A.A.M. Saleh and R.A. Valenzuela, "A statistical model for indoor multipath propagaton," IEEE Journal on Selected Areas in Communications, vol. SAC-5, pp. 128135, Feb. 1987.

[6] J.A. Högbom, "Aperture synthesis with a nonregular distribution of interferometer baselines," Astronomy and Astrophysics Supplement, vol. 15, pp. 417, 1974. 